\title{
Erratum to: Vortex Formations from Domain Wall Annihilations in Two-Component Bose-Einstein Condensates
}

\author{
Hiromitsu Takeuchi • Kenichi Kasamatsu • \\ Muneto Nitta $\cdot$ Makoto Tsubota
}

Published online: 24 October 2012

(C) Springer Science+Business Media New York 2012

\section{Erratum to: J Low Temp Phys (2011) 162:243-249 \\ DOI 10.1007/s10909-010-0294-0}

The first author's correct family name is Takeuchi.

The online version of the original article can be found under doi:10.1007/s10909-010-0294-0.

H. Takeuchi $(\bowtie) \cdot$ M. Tsubota

Department of Physics, Osaka City University, Sumiyoshi-Ku, Osaka 558-8585, Japan

e-mail: hiromitu@sci.osaka-cu.ac.jp

K. Kasamatsu

Department of Physics, Kinki University, Higashi-Osaka, Osaka 577-8502, Japan

\section{Nitta}

Department of Physics, and Research and Education Center for Natural Sciences, Keio University, Hiyoshi 4-1-1, Yokohama, Kanagawa 223-8521, Japan 